\title{
Spontaneous emission in non-local materials
}

\author{
Pavel Ginzburg ${ }^{1,2, \star}$, Diane J Roth ${ }^{1, \star}$, Mazhar E Nasir ${ }^{1, \star}$, Paulina Segovia ${ }^{1}$, Alexey V Krasavin ${ }^{1, \star}$, James Levitt ${ }^{1}$, \\ Liisa M Hirvonen ${ }^{1}$, Brian Wells ${ }^{3,4}$, Klaus Suhling ${ }^{1}$, David Richards ${ }^{1}$, Viktor A Podolskiy ${ }^{3}$ \\ and Anatoly V Zayats ${ }^{1}$
}

\begin{abstract}
Light-matter interactions can be strongly modified by the surrounding environment. Here, we report on the first experimental observation of molecular spontaneous emission inside a highly non-local metamaterial based on a plasmonic nanorod assembly. We show that the emission process is dominated not only by the topology of its local effective medium dispersion, but also by the non-local response of the composite, so that metamaterials with different geometric parameters but the same local effective medium properties exhibit different Purcell factors. A record-high enhancement of a decay rate is observed, in agreement with the developed quantitative description of the Purcell effect in a non-local medium. An engineered material non-locality introduces an additional degree of freedom into quantum electrodynamics, enabling new applications in quantum information processing, photochemistry, imaging and sensing with macroscopic composites.
\end{abstract}

Light: Science \& Applications (2017) 6, e16273; doi:10.1038/lsa.2016.273; published online 2 June 2017

Keywords: composite electromagnetic materials; non-local optical properties; plasmonic metamaterials; quantum electrodynamics; spontaneous emission

\section{INTRODUCTION}

When the behavior of a physical system at a given point depends on its state at another spatially separated region, the system is described as being non-local. Quantum states of light and matter are inherently non-local, reflecting the fundamental wave-particle duality ${ }^{1}$. Quantum entanglement is one of the most fascinating examples of non-localities in nature ${ }^{2,3}$. Its successful demonstration in optics with photons ${ }^{4,5}$ is enabled by inherently weak photon-photon interactions. Material systems, on the other hand, suffer from various decoherence effects, such as electron-electron, electron-phonon and other scattering mechanisms that virtually eliminate optical non-locality in homogeneous room-temperature media ${ }^{6}$. However, electromagnetic nonlocality may re-emerge in engineered composites, metamaterials ${ }^{7}$, where coherent surface plasmons mediate coupling between unit cells of artificial electromagnetic crystals.

Here, we analyze both experimentally and theoretically the process of spontaneous emission in a non-local environment using a plasmonic nanorod metamaterial platform that has been recently demonstrated to enable a topological transition between elliptic and hyperbolic dispersions. Experimentally, we demonstrate a broadband, macroscopically averaged lifetime reduction of the order of 30 for several fluorophores, while microscopic reductions in lifetime are orders of magnitude higher, as estimated from the experimental data. We develop new theoretical and numerical approaches capable of calculating the local density of photonic states in non-local media. Although non-local effects are known to have a weak impact on linear reflection and transmission through the metamaterial ${ }^{7}$, the situation is completely different in the quantum optical regime where non-locality results in an additional propagating mode inside the metamaterial, fundamentally altering the density of photonic states ${ }^{8}$. The dynamics of emitters' decay, and other quantum optical processes, inside a nanorod metamaterial are essentially dominated by the non-local response of the composite, as shown in this work.

The paper is organized as follows. In the Materials and methods section, we describe fabrication of the nanorod metamaterials and a flow-cell-based introduction of fluorophores inside the metamaterials. The details of fluorescence lifetime measurements are presented together with the lifetime distribution analysis based on the Laplace transform, which is paramount for understanding multi-exponential decay of an ensemble of emitters in a structured environment. This Laplace approach is verified by recovering the spontaneous emission lifetime distribution near a smooth Au film. The local and non-local models of the effective medium theory (EMT) for a composite metamaterial are then described. Subsequently, the analytical evaluation of the spontaneous emission lifetime is derived based on the EMT approach. Finally, the numerical model for the spontaneous emission inside the nanorod metamaterials is presented and compared with the EMT and experimental results. In the Results and discussion section,

\footnotetext{
${ }^{1}$ Department of Physics, King's College London, Strand, London WC2R 2LS, UK; ${ }^{2}$ Present address: School of Electrical Engineering, Tel Aviv University, Tel Aviv 69978, Israel; ${ }^{3}$ Department of Physics and Applied Physics, University of Massachusetts Lowell, One University Ave, Lowell, MA 01854, USA and ${ }^{4}$ Present address: Department of Physics, University of Hartford, West Hartford, CT 06117, USA

*These authors contributed equally to this work

Correspondence: P Ginzburg, Email: pginzburg@post.tau.ac.il

Received 10 June 2016; revised 28 November 2016; accepted 13 December 2016; accepted article preview online 19 December 2016
} 
the mode structure of the metamaterials is derived in the local and non-local EMT approximations. The role of non-locality and losses is elucidated on the topology of the isofrequency surfaces in elliptic and hyperbolic regimes of dispersion. Spontaneous emission rates are evaluated for the emitters inside the composite in the local and nonlocal EMT descriptions. The dependence of the mode dispersion and the photoluminescence (PL) lifetime on the scaling of the elementary cell of the nanorod composite is then shown, which is absent in the local EMT description and is a purely non-local effect. These analytical results are confirmed with full-vectorial numerical simulations. The spontaneous emission lifetimes of different fluorophores emitting at different wavelengths, falling within elliptical, epsilon-near-zero (ENZ), and hyperbolic dispersion regimes of the metamaterial are measured and analyzed. The results are in excellent agreement with the numerical solutions of Maxwell equations and analytical model, confirming an important role of non-locality for spontaneous emission inside the metamaterial.

\section{MATERIALS AND METHODS}

\section{Metamaterial fabrication}

Plasmonic nanorod metamaterials were fabricated by Au electrodeposition into highly ordered nanoporous anodic alumina oxide (AAO) templates on glass cover slips. An Al film of $700 \mathrm{~nm}$ thickness was deposited on a substrate by magnetron sputtering. The substrate comprised a glass cover slip with a $10 \mathrm{~nm}$ thick adhesive layer of tantalum pentoxide and a $7 \mathrm{~nm}$ thick $\mathrm{Au}$ film, acting as a weakly conducting layer. Highly ordered, nanoporous AAO was synthesized by a two step-anodization in $0.3 \mathrm{M}$ oxalic acid at $40 \mathrm{~V}$. After an initial anodization process, the formed porous layer was partly removed by etching in a solution of $\mathrm{H}_{3} \mathrm{PO}_{4}(3.5 \%)$ and $\mathrm{CrO}_{3}\left(20 \mathrm{~g} \mathrm{~L}^{-1}\right)$ at $70{ }^{\circ} \mathrm{C}$, which resulted in an ordered pattern. At the next step, the sample was anodized again under the same conditions as in the first step. The anodized AAO was subsequently etched in $30 \mathrm{mM} \mathrm{NaOH}$ to achieve pore widening. Gold electrodeposition was performed with a three electrode system, using a non-cyanide solution. The length of nanorods was controlled by the electrodeposition time. Free standing gold nanorod metamaterials were obtained after dissolving the nanoporous alumina template in a mix solution of $0.3 \mathrm{M} \mathrm{NaOH}$ and $99.50 \%$ ethanol. The nanorod array parameters used in the reported experiments are $50 \pm 2 \mathrm{~nm}$ diameter, $100 \mathrm{~nm}$ period and $250 \pm 5 \mathrm{~nm}$ height (Figure 1a). The optical properties and the extinction spectra of the metamaterial for various illumination angles are shown in Figure 1b-1d, respectively.

\section{Fluorescence lifetime measurements}

Four dye molecules, D1 (fluorescein), D2 (Alexa 514), D3 (ATTO 550 ) and D4 (ATTO 647N) with the emission wavelengths 514, 550, 575 and $670 \mathrm{~nm}$, respectively, were used to probe local density of states of the metamaterial. For D1 and D2 dyes, the solvent was ethanol and the concentration was $2.5 \times 10^{-5} \mathrm{~mol} \mathrm{~L}^{-1}$. For D3 and D4 dyes, the solvent was made of $10 \mathrm{mM}$ of Tris-HCL, $1 \mathrm{mM}$ of EDTA, $30 \mathrm{mM}$ of $\mathrm{NaCl}$ and $\mathrm{NaOH}$ to adjust the $\mathrm{pH}$ of the solution to 7.85 , and the concentration of $10^{-6} \mathrm{~mol} \mathrm{~L}^{-1}$ was chosen. The dye solutions were introduced in the metamaterial using a flow cell, which was positioned on a confocal microscope for fluorescence measurements. In order to ensure a correct comparison between the lifetime measurements for different emitters, the same metamaterial was used for the measurements of all four dyes. For repeated measurements, the dyes' solution was washed out and the sample was plasma cleaned before changing emitters.
Time-resolved PL measurements were performed using timecorrelated single photon counting (TCSPC) ${ }^{9}$ based on a SPC-150 (Becker-Hickl) system. The wavelength of the TM-polarized excitation light from a Fianium super-continuum laser $(<10$ ps pulse duration, $20 \mathrm{MHz}$ repetition rate) was selected (525 nm for ATTO 550, $633 \mathrm{~nm}$ for ATTO 647N, $470 \mathrm{~nm}$ for Alexa 514 and $470 \mathrm{~nm}$ for fluorescein). The excitation light was focused on the sample with a $100 \times$, numerical aperture $=1.49$ oil-immersion objective and the resulting PL signal collected by the same objective. Appropriate band-pass filters centered on the peak emission wavelength of each dye were used to collect the PL signal and prevent any laser contribution to the measured light. For each dye, three sets of measurements were carried out on a metamaterial, a thin $(50 \mathrm{~nm})$ gold film, and a glass substrate. The PL spectra of the different dyes on glass and inside the metamaterial are shown in Supplementary Fig. S1.

\section{Fluorescence lifetime data analysis}

In order to analyze the time-dependence PL data, the inverse Laplace transform was used ${ }^{10}$, which does not rely on any preliminary assumption on emission process and adequately reproduces the distribution of the lifetimes present in the experimental data. This procedure is implemented by solving the integral equation

$$
I(t)=\int_{0}^{\infty} F(s) e^{-s t} \mathrm{~d} s
$$

where $I(t)$ is the time dependent fluorescence intensity (measured quantity), deconvoluted from the instrumental response function (measured in a separate experiment), and $F(s)$ is the relative weight of different single exponential decay components. The deconvolution was performed by applying Tikhonov regularization technique, also introducing low pass filtering, reducing the noise of the decay tail. Since the inverse Laplace transform is known to be an ill-defined problem (especially for analysis of noisy data), an iterative fitting procedure was applied in order to achieve stable results. This type of analysis assumes that the fluorophores are in the weak coupling regime of interaction with the surrounding environment.

\section{Lifetime analysis of the ensemble of emitters near a gold film}

A semi-analytical expression for decay rate modification of a point-like emitter near an interface between two dielectric materials relies on the knowledge of the electromagnetic Green's function ${ }^{11}$ and its classical quantum correspondence to the spontaneous emission rate (proportionality to the imaginary part of the Green's function $)^{12,13}$. Hence, given the lifetime distributions of fluorophores near a glass substrate $F_{\text {Glass }}^{\text {exp }}(s)$, the corresponding distribution near a gold film can be derived as

$$
F_{\text {Au }}^{\text {Theor }}(s) \sim \int_{d_{\text {min }}}^{d_{\text {max }}}\left|\vec{E}_{\text {pump }}(z)\right|^{2} Q_{\text {loc }}(z) n(z) F_{\text {Glass }}^{\text {exp }}(s / P(z)) \mathrm{d} z,
$$

where $P(z)$ is the position dependent polarization-averaged Purcell factor near the Au surface, $\left|\vec{E}_{\text {pump }}(z)\right|^{2}$ is the position-dependent intensity of the excitation light, $Q_{\mathrm{loc}}(z)$ is the fluorophore local quantum yield, and $n(z)$ is the distribution density of the fluorophores along the focal depth, which was assumed to be uniform. The depth of focus $\left(d_{\text {max }}-d_{\text {min }}\right)$ was taken to be $\sim 175 \mathrm{~nm}$ for the best fit to the experimental data. Slight deviations between the fitting and experiment may arise due to sensitivity of the Laplace transform to noise in experimental data, and the assumption on $n(z)$, which in principle, may encapsulate stochastic inhomogeneity of the solution. 
a
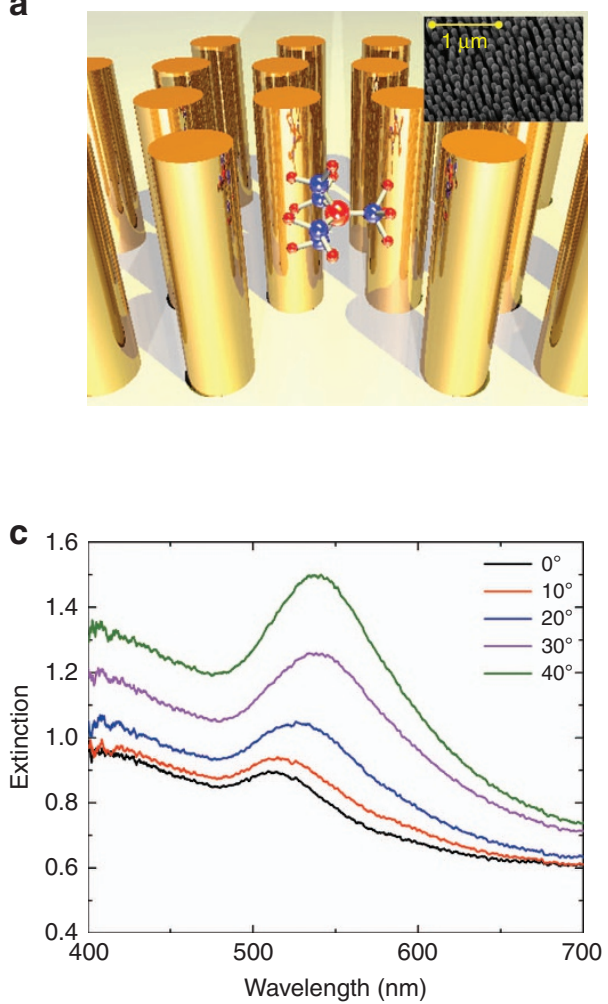

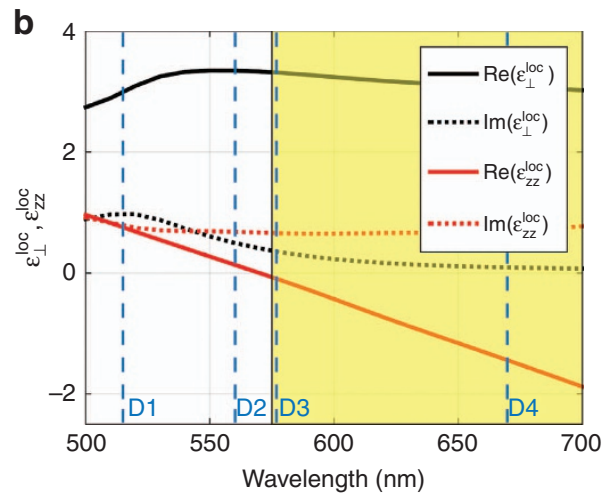

d

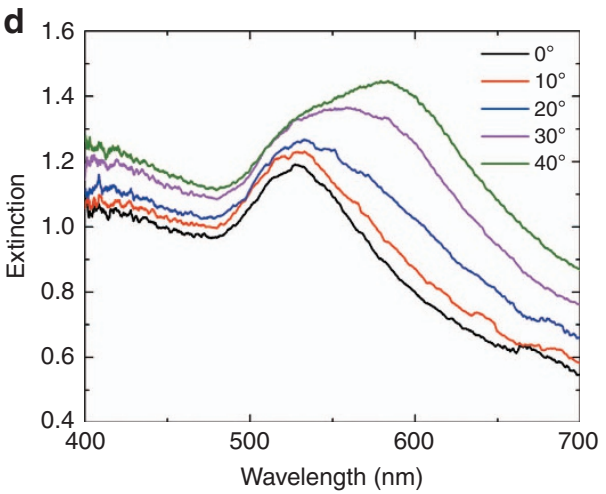

Figure 1 (a) Schematics of a nanorod metamaterial with a fluorescent molecule inside. Inset shows SEM image of the metamaterial comprised of Au nanorods in air (scale bar is $1 \mu \mathrm{m}$ ). (b) Local effective medium parameters of the metamaterials (Au nanorods in ethanol) determined from the geometry of the nanorod metamaterial $50 \pm 2 \mathrm{~nm}$ diameter, $100 \mathrm{~nm}$ period and $250 \pm 5 \mathrm{~nm}$ height) which reproduce the experimental extinction spectra in c. Vertical dashed lines indicate the emission wavelengths of the dyes for the PL lifetime measurements. (c, d) Extinction spectra of the metamaterials consisting of free standing nanorods for different angles of incidence of $\mathrm{p}$-polarized light in $\mathbf{c}$ air and $\mathbf{d}$ ethanol.

Effective medium models of the metamaterial

For light wavelengths much larger than all the characteristic sizes of the composite, such as nanorod diameter and their period, the optical properties of the metamaterial can be described with the effective permittivity, which can be used to calculate transmission, reflection and absorption spectra of the composite. Due to symmetry, the optical properties of a nanorod metamaterial resemble those of an homogeneous uniaxial anisotropic medium with optical axis parallel to the nanorods ( $z$-direction in Figure 1b). Macroscopically, these optical properties are described by a diagonal permittivity tensor $\hat{\epsilon}$ with $\epsilon_{\mathrm{xx}}=\epsilon_{\mathrm{yy}} \equiv \epsilon_{\perp} \neq \epsilon_{z z}$. In analytical calculations, a layer of a metamaterial was represented as a homogeneous layer described by either local ${ }^{14}$ or non-local ${ }^{15}$ effective medium theories.

Local EMT. In a conventional, local effective medium description, the permittivity of the metamaterial is given by the Maxwell Garnett theory:

$$
\epsilon_{\perp}^{\mathrm{mg}}=\epsilon_{h} \frac{(1+p) \epsilon_{\mathrm{Au}}+(1-p) \epsilon_{\mathrm{h}}}{(1+p) \epsilon_{\mathrm{h}}+(1-p) \epsilon_{\mathrm{Au}}} ; \epsilon_{z z}^{\mathrm{mg}}=p \epsilon_{\mathrm{Au}}+(1-p) \epsilon_{\mathrm{h}}
$$

with $\epsilon_{\mathrm{h}}$ and $\epsilon_{\mathrm{Au}}$ being the permittivities of the host material and gold, respectively, and $p=\pi(r / a)^{2}$ is the fill factor of the metal inside the matrix ( $r$ is the nanorod radius and $a$ is the period of the array). The Drude model, with corrections for restricted mean free path of electrons ${ }^{16}$, previously shown to be in quantitative agreement with experimental results ${ }^{7,16}$, was used to describe the permittivity of electrochemically deposited gold. The spectral behavior of the components of effective permittivity tensor of the metamaterial used in the experiments is shown in Figure 1b.

Non-local EMT. When optical losses in Au nanorods are small, a local effective medium description of metamaterial becomes invalid, and a more complex, non-local effective medium response must be considered $^{7}$. In non-local EMT, the components of the permittivity tensor perpendicular to the optical axis are still described by the Maxwell Garnett theory $\left(\epsilon_{\mathrm{xx}}=\epsilon_{\mathrm{yy}}=\epsilon_{\perp}^{\mathrm{MG}}\right)$, while the component of the permittivity tensor along the optical axis becomes explicitly dependent on the wave vector,

$$
\epsilon_{z z}\left(k_{z}\right)=\xi\left(k_{z}^{2} \frac{c^{2}}{\omega^{2}}-\left(n_{z}^{1}\right)^{2}\right) ; \xi=p \frac{\epsilon_{\mathrm{Au}}+\epsilon_{\mathrm{h}}}{\epsilon_{\mathrm{h}}-\left(n_{\infty}^{1}\right)^{2}}
$$

where $n_{z}^{1}$ is the effective refractive index of the cylindrical surface plasmons that propagate in a nanorod composite with the nanorod permittivity $\epsilon_{\mathrm{Au}}$ and $n_{\infty}^{1}$ represents the limit of $n_{z}^{1}$ for perfectly conducting nanorods ${ }^{17}$. These parameters can be calculated either numerically or by solving an eigenvalue-type problem.

The transfer matrix method ${ }^{17}$, was used to calculate transmission and reflection of a planar slab of the metamaterial for a given excitation frequency and angle of incidence.

The effective medium model adequately describes the extinction spectra observed in experiment (cf. Figure $1 \mathrm{c}$ and $1 \mathrm{~d}$ and Supplementary Fig. S2). The two spectral features, seen in extinction spectra at $\lambda=525 \mathrm{~nm}$ (for all incident angles) and at $\lambda=575 \mathrm{~nm}$ (for larger incident angles) correspond to the Maxwell Garnett 
resonance $\left(\epsilon_{\perp}^{\mathrm{mg}} \gg 1\right)$ and the ENZ regime $\left(\epsilon_{z z}^{\mathrm{mg}} \simeq 0\right)$, respectively. For very long wave vectors that approach the edge of the Brillouin zone of the nanorod array, both local and non-local EMTs break down and should not be used. Similar behavior was recently observed in layered composites when the wave vector in the direction perpendicular to the layers becomes comparable to the period ${ }^{15}$.

\section{Fluorescence decay rate calculations from EMT formulation}

A Green's function formalism was used to analyze the emission rate modification ${ }^{18}$. In this approach, the emission rate is proportional to the imaginary part of the Green's function, representing the electric field $\vec{E}$ generated by the point dipole $\vec{d}$ at the location of the dipole $8,11,18$

$$
\frac{\Gamma}{\Gamma_{0}} \simeq \frac{3 c^{3 \operatorname{Im}}(\vec{E} \cdot \vec{d})}{2 \omega^{3} \quad\left|W_{0}\right|^{2}}
$$

where $\Gamma_{0}$ is the emission rate in the free space, while $\Gamma$ is the rate modified due to surrounding environment, and $W_{0}$ is the power radiated by the dipole in free space, which serves as a normalization constant. $\hat{z}$ - polarized and in-plane polarized dipoles were considered separately, and the total emission rate was calculated as the weightedaverage of the two dipole directions. This assumption is related to a completely random distribution of dye molecules inside the solution. The transfer matrix formalism was used to take into account the effect of multiple reflections of the dipole's emission inside the composite layer ${ }^{19}$. In order to avoid singularities caused by placing the dipole inside a lossy medium, the dipole was placed inside a $1 \mathrm{~nm}$ thin layer of lossless medium, cut out inside the lossy metamaterials (the technique known as extraction of a depolarization volume). In the non-local case, two TM-polarized modes were considered as independent emission channels; however, the obtained results suggest that the hyperbolic-like mode dominates emission in both elliptic and hyperbolic regimes, similar to what has been previously reported in emission inside an infinite idealized metamaterial ${ }^{20}$.

\section{Fluorescence decay rate calculation from numerical modeling}

Numerical modeling was performed using the finite element method (FEM) implemented in Comsol Multiphysics software. The Purcell factor at various locations inside the nanorod metamaterial was calculated as a ratio of power flow from a point dipole placed at a given position and the corresponding value for a dipole in vacuum. In order to cross-check reliability of the numerical results, two independent methods were used for the power flow estimation: 1) via a Poynting vector flux through a small sphere ( $5 \mathrm{~nm}$ radius) enclosing the point dipole and 2) via the energy dissipation rate of the dipole, $W(\vec{r})=-\frac{1}{2} \operatorname{Re}\left[\vec{E}^{*}(\vec{r}) \cdot \vec{J}(\vec{r})\right]$, where $\vec{E}(\vec{r})$ is the electric field produced by the dipole at the point of its location and $\vec{J}(\vec{r})$ is the dipole current. Both methods showed excellent agreement with each other. To take into account the arbitrary orientation of an emitting dipole, the power flow was calculated separately for emitters aligned with each coordinate axis, and the overall enhancement was calculated as the average $\Gamma(\vec{r})=\frac{1}{3} \Gamma_{x}(\vec{r})+\frac{1}{3} \Gamma_{y}(\vec{r})+\frac{1}{3} \Gamma_{z}(\vec{r})^{21}$. The results of these classical electromagnetic calculations can be directly mapped onto the quantum decays of emitters, by employing the classical quantum correspondence of radiation reaction forces ${ }^{18}$. In order to simulate an infinite number of nanorods in the metamaterial, the number of rods in the finite-size arrays was gradually increased with periodic boundary conditions on the sides of the nanorod patch. The convergence of the Purcell factor with the number of the nanorods within the simulation domain (Supplementary Fig. S3) confirms that a $10 \times 10$ nanorod array with periodic boundary conditions can be used to analyze the behavior of a dipole in infinite metamaterial. The averaging over the dipole position within the unit cell of the array of the metamaterial was performed, assuming a uniform distribution of the emitters with a position-dependent decay rate and local excitation efficiency, by an excitation light illuminating the metamaterial from the substrate side: $I(t) \propto \int_{e-c}\left|\vec{E}_{\text {pump }}(\vec{r})\right|^{2} Q_{\text {loc }}(\vec{r}) n(\vec{r}) \exp \left(-P(\vec{r}) \cdot \Gamma_{0} t\right) d^{3} \mathrm{r}$, where the integration is performed over the dye-filled volume within the elementary cell $(\mathrm{e}-\mathrm{c})$, taking into account the distance dependent number of emitters $n(\mathbf{r})$. Q loc $(\mathbf{r})$ is the local quantum efficiency defining how much of the emitted power is measured in the farfield (the internal quantum yield of the emitters was taken to be 1 in all the simulations). The local pump intensity was found to vary by only $8 \%$ between 470 and $633 \mathrm{~nm}$ excitation wavelength used in the experiment and was averaged for these values. Since this equation yields a multi-exponential decay, a procedure equivalent to the inverse Laplace transform was applied in order to recover the theoretically predicted lifetime distribution, in a manner similar to analysis of the experimental measurements.

\section{RESULTS AND DISCUSSION}

\section{Electromagnetic waves in the metamaterial and topology of isofrequency surfaces}

Homogeneous anisotropic media support the propagation of two types of waves that differ by their polarization. Ordinary waves (TE modes) that have electric field normal to the metamaterial's optical axis $\vec{E} \perp \hat{z}$ (Figure 1a) do not experience an anisotropy of dielectric permittivity. On the other hand, extraordinary waves (TM modes) that have magnetic field $\vec{H} \perp \hat{z}$ are strongly affected by material anisotropy. The dispersion of the extraordinary waves, the relationship between components of the wave vector $\vec{k}$, angular frequency $\omega$, and speed of light $c$, is given by

$$
\frac{k_{\mathrm{x}}^{2}+k_{\mathrm{y}}^{2}}{\epsilon_{z z}}+\frac{k_{\mathrm{z}}^{2}}{\epsilon_{\perp}}=\frac{\omega^{2}}{c^{2}}
$$

The isofrequency surface, which is defined as the locus of points with coordinates $\left\{k_{\mathrm{x}}(\omega), k_{\mathrm{y}}(\omega), k_{\mathrm{z}}(\omega)\right\}$, forms either an ellipsoid or a hyperboloid, depending on the relationship between the signs of $\epsilon_{\perp}$ and $\epsilon_{z z}$ which, in turn, are wavelength-dependent. The geometrical properties of this isofrequency surface are often referred to as the optical topology of a metamaterial ${ }^{22}$. The effective permittivity of the metamaterials used in this study (Figure 1b) shows that the composite operates in the elliptic regime for wavelengths below the $575 \mathrm{~nm}$, exhibits ENZ response at around $575 \mathrm{~nm}$, corresponding to the effective plasma frequency ${ }^{23}$ and operates in the hyperbolic regime for longer wavelengths. Optical topology predicted by Equation (6) exhibits typical ellipsoidal isofrequencies for TE modes in both elliptic and hyperbolic regimes (Figure 2a and 2b). The dispersion of TM modes in the hyperbolic regime is described by a hyperboloid (Figure 2d). Dispersion of TM modes in the elliptic regime is strongly affected by material losses ( $c f$. Supplementary Fig. S4g-i) and represents an ellipsoid in the center of the Brillouin zone which is increasingly deformed for higher wavenumbers (Figure 2c).

The optical topology of metamaterials has a marked effect on their quantum optical properties, leading, theoretically, to a singularity in the local density of optical states in homogeneous hyperbolic media ${ }^{24}$ (this effect is limited by granularity in metamaterials ${ }^{8}$ ). The density of optical states affects the rate of spontaneous emission ${ }^{25}$, nonradiative energy transfer between molecules ${ }^{26}$, and other processes. Recent 
a
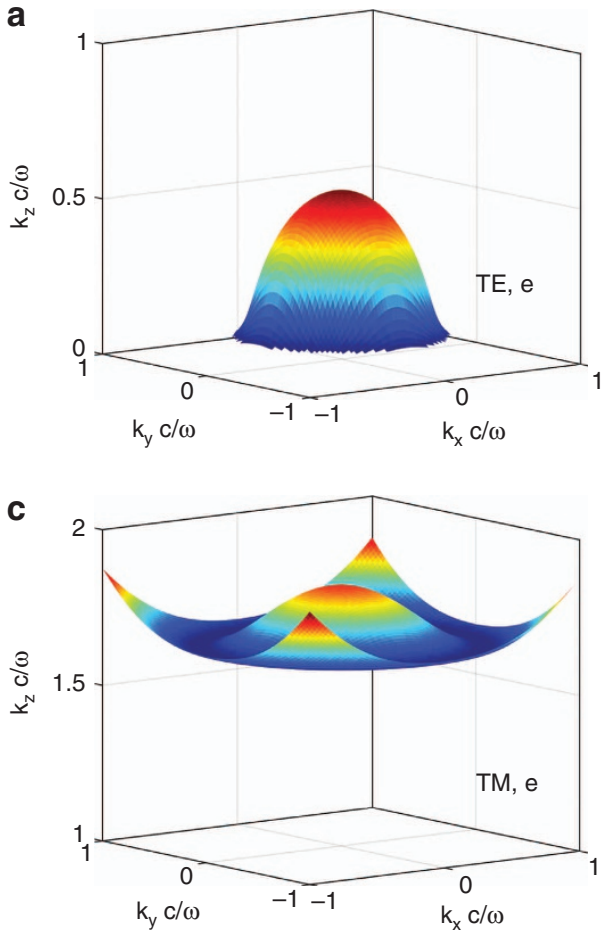

b

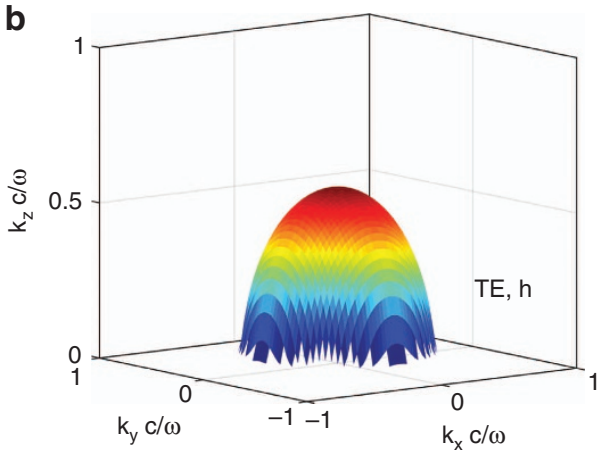

d

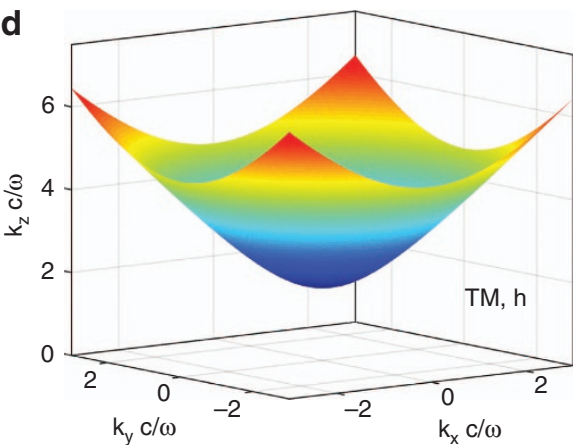

Figure 2 Isofrequency surfaces describing the dispersion of the propagating modes in the metamaterial with parameters as in Figure $1 \mathrm{~b}$, calculated with the local EMT: (a, c) elliptic (denoted e) regime $(\lambda=550 \mathrm{~nm}),(\mathbf{b}, \mathbf{d})$ hyperbolic (denoted $\mathrm{h}$ ) regime $(\lambda=650 \mathrm{~nm})$ for $(\mathbf{a}, \mathbf{b})$ TE- and (c, d) TM-polarized modes. The non-monotonic behavior of the TM-polarized mode in the elliptical regime (c) is a direct consequence of the material absorption (Supplementary Fig. S2).

experimental studies, mostly focused on emitters near a layered metamaterial design, have demonstrated relatively modest 5-10 fold enhancements of the decay rate in the hyperbolic dispersion case and smaller enhancements in the elliptic dispersion regime ${ }^{22}$. Similar results were reported for emitters on top of hyperbolic plasmonic nanorod metamaterials ${ }^{27}$. Magnetic hyperbolic metamaterials provided a possibility to probe magnetic-dipole transitions ${ }^{28}$. However, the geometry of these previous studies prevented a test of emission dynamics in the non-local metamaterial regime, most pronounced inside nanorod metamaterials operating in the elliptical regime $e^{7,17,29,30}$ (corresponding to $\lambda<575 \mathrm{~nm}$ for the metamaterials used in this work).

\section{Optical non-localities in nanorod media}

There exists a frequency range in which the nanorod metamaterial supports the propagation of not two, but three different modes ${ }^{7,30}$. One of these modes is a TE-polarized ordinary wave (Figure $3 \mathrm{a}$ and $3 \mathrm{~b}$ ), while the other two waves have identical (TM) polarization, but different effective indices. The dispersion of TM-polarized waves is given by

$$
\left(k_{\mathrm{z}}^{2}-\left(n_{z}^{1}\right)^{2} \frac{\omega^{2}}{c^{2}}\right)\left(k_{\mathrm{z}}^{2}-\epsilon_{\perp}^{\mathrm{mg}} \frac{\omega^{2}}{c^{2}}\right)=-\frac{\epsilon_{\perp}^{\mathrm{mg}} \omega^{2}}{\xi c^{2}} k_{\mathrm{x}}^{2}
$$

The topology of these waves is shown in Figure $3 c$ and $3 \mathrm{~d}$ ). Comparing Figure 2 and Figure 3, it is seen that while in the hyperbolic regime the differences are minimal, the behavior in the elliptic regime is drastically different. The isofrequency of one of the TM polarized waves now qualitatively agrees with an ellipsoid, predicted by Equation (6). The second, additional TM polarized mode which is absent in the local effective medium theory, has a hyperboliclike topology (Figure 3c).
Transmission spectra of the slab of the metamaterial, calculated with the local and non-local permittivity models for the metamaterial parameters used in this work, are almost identical with small deviations at the larger angles of incidence (Supplementary Fig. S2), with the non-local EMT providing better correspondence to the shape of the experimentally measured spectra (Figure 1c and 1d). Nevertheless, there is a drastic disagreement between predictions of the two approaches for the evaluation of emission properties of the dipole embedded inside the metamaterial. The additional wave described in the non-local EMT represents a collective excitation of cylindrical surface plasmons propagating on metallic nanorods ${ }^{17}$. This mode is a metamaterial analog of collective light-matter excitations, excitonpolaritons, that are known to enable additional waves in homogeneous semiconductors $^{6}$. In both homogeneous and composite media, properties of additional waves can be successfully described by introducing non-locality, that is, a wave vector dependence of the permittivity of the material. However, although the permittivity of homogeneous media is essentially fixed by nature, the electromagnetic response of composites can be engineered.

\section{Engineering non-local response by scaling metamaterial's unit cell} and losses

The dispersion of modes in plasmonic nanorod composites can be controlled by scaling the unit cell, a process that does not change the local effective medium response, but does affect the non-local one. The predicted changes in the dispersion of optical modes as a result of geometry scaling are summarized in Figure $3 \mathrm{e}$ and $3 \mathrm{f}$. Material losses can be controlled by the choice of plasmonic metal or by fabrication (for example, annealing) and serve as an additional degree of freedom for engineering the optical properties of a metamaterial. 

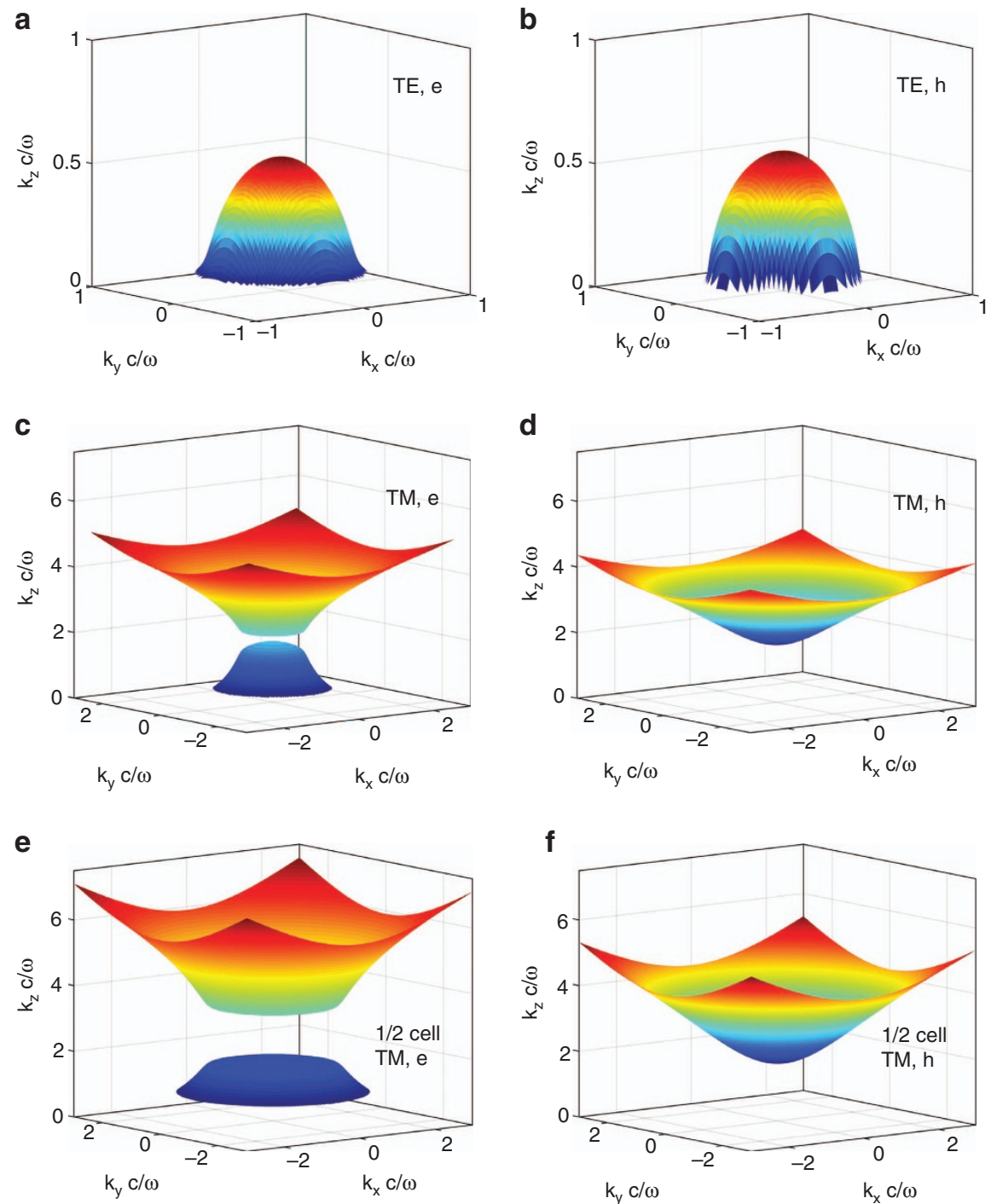

Figure 3 Dispersion of the modes supported by the nanorod metamaterial calculated using the non-local effective medium theory: (a, c, e) elliptic (denoted e) regime $(\lambda=550 \mathrm{~nm})$ and $(\mathbf{b}, \mathbf{d}, \mathbf{f})$ hyperbolic (denoted $\mathrm{h})$ regime $(\lambda=650 \mathrm{~nm})$ in the case of $(\mathbf{a}, \mathbf{b})$ TE- and $(\mathbf{c}-\mathbf{f})$ TM-modes for $(\mathbf{a}-\mathbf{d})$ the metamaterial considered in experiments (as in Figure $1 \mathrm{~b}$ ) and (e, f) metamaterial with $50 \%$ unit cell and nanorod diameter ( $25 \mathrm{~nm}$ diameter, $50 \mathrm{~nm}$ period). The scaling does not change the local effective medium parameters of the composite (Figure 2a-d).

The dispersion of the modes supported by the nanorod composite, predicted by local and non-local effective medium theories clearly shows that dispersion of TE polarized modes does not significantly depend on materials absorption or unit cell size and is close to those presented in Figure $2 \mathrm{a}$ and $2 \mathrm{~b}$. On the other hand, when the metamaterial operates in the elliptical regime, absorption, along with unit cell configuration, plays an important role in defining the topology of TM-polarized modes (Supplementary Fig. S4). When losses are small, the two TM polarized modes represent well-separated branches in the wave vector space, with the lower branch having elliptical behavior and the upper branch exhibiting hyperbolic-like dynamics (Supplementary Fig. S4a and S4d). As the unit cell becomes smaller, the behavior of the elliptic branch approaches predictions of the local effective medium theory (Supplementary Fig. S4g), while the upper branch moves 'up', increasing the effective index of the mode. As losses increase, the two TM-polarized modes approach each other (Supplementary Fig. S4b and S4c). Scaling the size of the unit cell of metamaterial still drastically affects the topology of these waves (Supplementary Fig. S4e and S4f). Note that when losses are high, the local EMT converges to the upper (hyperbolic) wave (Supplementary Fig. S4i); however, in the regime of moderate losses, realized in our work, the local EMT becomes invalid, predicting the response that does not match the properties of either of the two modes of the metamaterial (Supplementary Fig. S4h). When the metamaterial operates in the hyperbolic regime, the elliptical branch of the TMdispersion cuts off, and the propagation of light is dominated by the hyperbolic branch (Supplementary Fig. S5). The effect of the spatial dispersion is now essentially limited to a quantitative correction of the dispersion. Decreasing the unit cell size or reducing losses causes the dispersion to approach a 'perfect' hyperboloid, while decreasing the unit cell alone causes the response to approach predictions of the local effective medium theory.

From the point of view of a quantum emitter, an additional electromagnetic wave represents a separate emission decay channel 
that, in turn, affects its radiation dynamics, and drastically increases the density of optical states in the elliptic regime.

\section{Experimental studies}

The analysis of decay dynamics of four fluorescent dyes with different emission wavelengths spanning elliptic and hyperbolic regimes and placed inside the metamaterial is summarized in Figure 4. Although all the studied emitters show nearly single-exponential fluorescence decays in a homogeneous environment, the presence of plasmonic surfaces and nanostructures makes the dynamics multi-exponential. This effect does not represent the strong coupling regime of interaction ${ }^{31}$, but rather is the direct result of many emitters contributing to the signal, with each individual emitter having its own position- and polarizationdependent decay rate in the structured environment.

Here we have used the inverse Laplace transform ${ }^{10}$, which does not rely on any preliminary assumptions and provides the distribution of the lifetimes present in the observations. Emitters situated above a glass substrate show smooth localized lifetime distributions peaked around their standard values of a few nanoseconds (Figure 5). The comparison of emission for glass and $\mathrm{Au}$ interfaces reveals the expected behavior, caused by the presence of the metal, and related plasmonic excitations ${ }^{11}$. Once the dependence of the lifetime on the emitter's spatial position is taken into account, an almost perfect agreement between theoretical predictions and experimental measurements of lifetime distributions is achieved for the emitters near the metal interface (Figure 5). The overall peak to peak rate enhancement is less than 2. For example, the lifetime distribution for D1 emitters (Figure 5a) is peaked at $3.9 \mathrm{~ns}$, representing the reduction of the macroscopically averaged lifetime by a factor of $\sim 1.2$. Spatial averaging takes into account the random distribution of molecules inside the sample volume. The excellent agreement between the experimental data and the model indicates that, various possible collective effects which could affect the dynamics, such as super-fluorescence ${ }^{32,33}$, can be ruled out.

The dynamics measured for the emitters inside the metamaterial exhibits even faster decay with an even broader distribution (7-100 times) of the lifetimes than for a single metal interface (Figure 5 and Supplementary Fig. S3). The span of the lifetime distributions reflects a strong position-dependent decay rate enhancement for the emitters inside the nanorod array unit cell ${ }^{34}$. Substantial lifetime reduction was observed for all wavelengths, possibly limited by the instrument function of the measurements.

The spectral dependence of the enhancement of the emission rates observed in experiments and obtained from direct numerical solutions of the Maxwell equations have similar features of a broad spread of the lifetimes at all emission wavelengths and weak wavelength dependence a
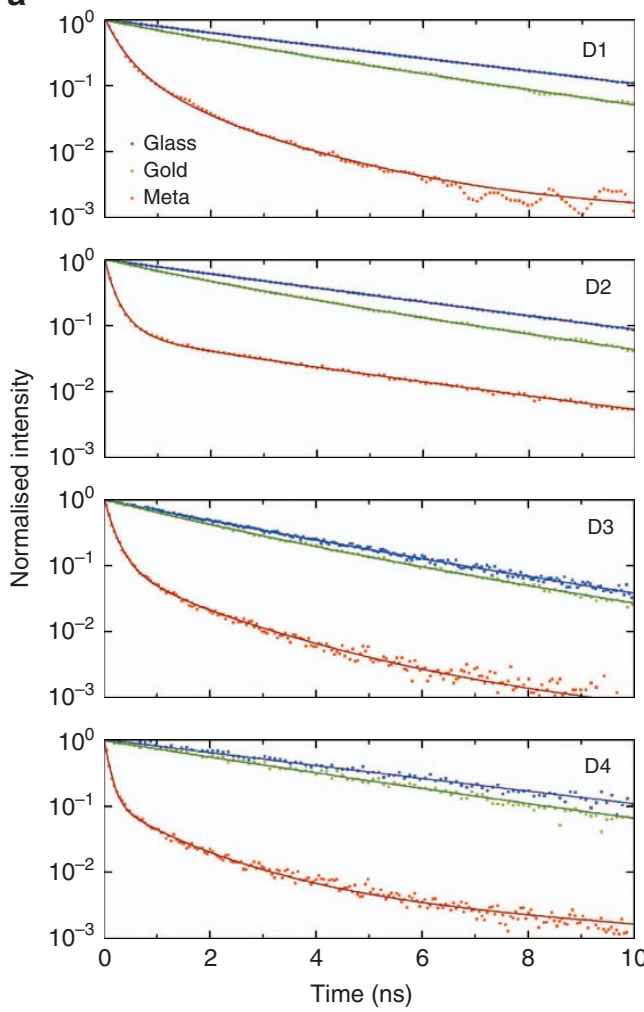

b

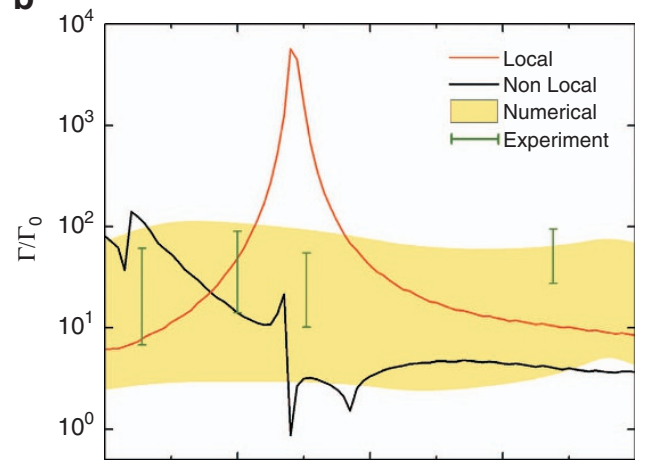

C

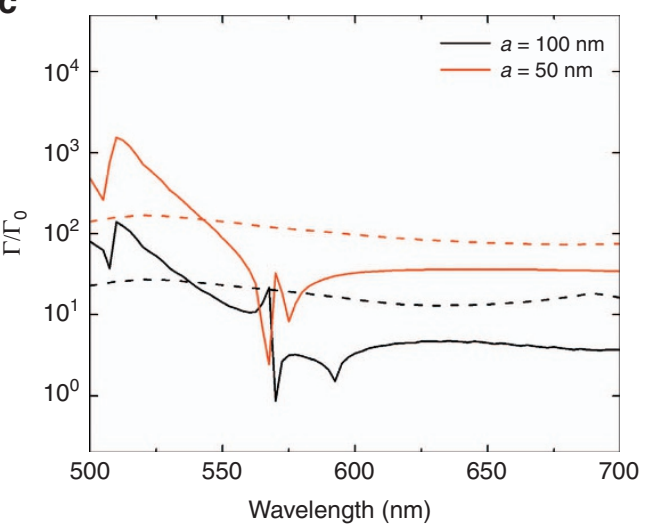

Figure 4 (a) Fluorescence dynamics of the emitters with the emission wavelength in different dispersion regimes of the metamaterial: (blue) on a glass surface, (green) on an Au film, (red) inside the metamaterial. The studied emitters are D1 (fluorescein), D2 (Alexa 514), D3 (ATTO 550) and D4 (ATTO $647 \mathrm{~N}$ ) with the emission wavelengths $514 \mathrm{~nm}, 550 \mathrm{~nm}, 575 \mathrm{~nm}$ and $670 \mathrm{~nm}$, respectively. Solid lines present dynamics recovered by applying an inverse Laplace transform to the experimental data (dots). (b) Spectral dependence of the lifetime averaged over the dipole orientation: (red) local theory, (black) non-local theory, (bars) experimental data corresponding to the width of the lifetime distribution at $10 \%$ of the modal amplitude (Figure 5), (shaded area) the width of the simulated lifetime distribution at $10 \%$ of the modal amplitude obtained applying the inverse Laplace transform to the decay curves after the averaging over the dipole position within the elementary cell of the metamaterial (Supplementary Fig. S3a), (c) Spectral dependence of the rate enhancement obtained with the non-local theory (solid lines) and the full-wave numerical modeling at one position within the metamaterial (dashed lines, position 3 in Supplementary Fig. S3a): (black) the metamaterial as in b used in the experiments ( $d=50 \mathrm{~nm} ; a=100 \mathrm{~nm})$ and (red) the metamaterial with $50 \%$ unit cell $(d=25 \mathrm{~nm} ; a=50 \mathrm{~nm})$ that yields an identical local effective medium response. 

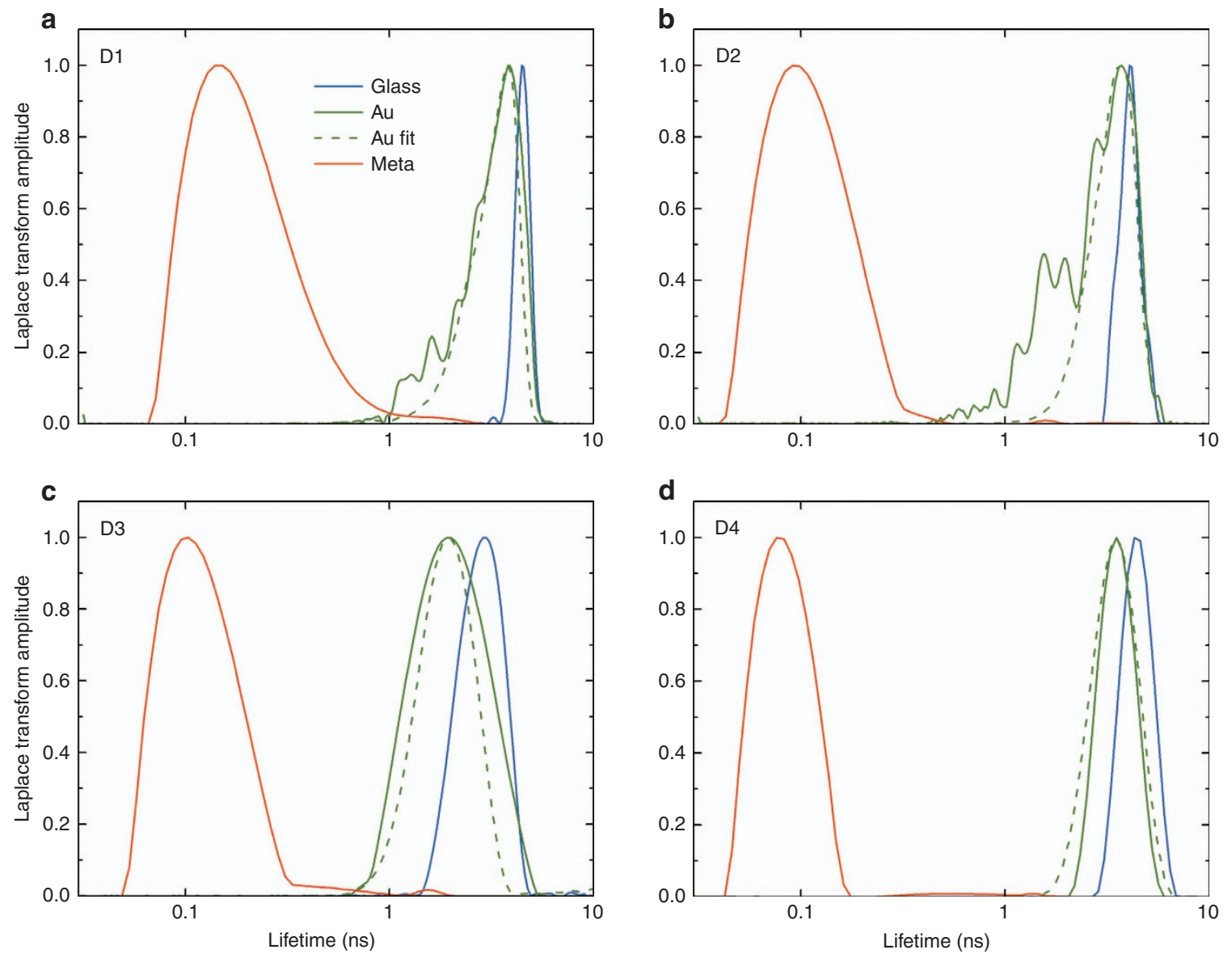

Figure 5 Experimental fluorescence lifetime (1/s) distribution of the emitters in different environments extracted using a Laplace transform method (Equation (1)) from the time-resolved PL measurements: (blue) glass substrate, (green) Au film, and (red) inside the metamaterial. Dashed green lines represent the lifetime distributions above the smooth Au surface recalculated from the measured lifetime distributions in the dielectric environment shown with blue lines. (a) D1: Fluorescein, (b) D2: Alexa 514, (c) D3: ATTO 550 and (d) D4: ATTO 647N.

of this distribution (Figure $4 \mathrm{~b}$ ). The experimental data show that the distribution of decay rate enhancements, from 10 to 100 (for the high and low-lifetime cut-offs, respectively, at $10 \%$ of the modal lifetime contribution), is almost independent of the wavelength across the elliptic, ENZ and hyperbolic regimes (Figure 4b). Local EMT predicts a strong enhancement of the decay rate in the ENZ regime, in sharp contrast with the observed behavior. At the same time, the experimental data are in closer agreement with predicted wavelength dependence of the decay rate given by non-local EMT. The remaining quantitative difference between the predictions of non-local effective medium theory (that averages the response of the composite) and experimental data stems from strong dependence of density of optical states on the position of the dipole within the unit cell ${ }^{10}$. Numerical modelling of a dipole emission inside the nanorod composites (Supplementary Fig. S3) confirms this position dependence and is also in agreement with the experimental data and the non-local theory. It should be noted that neither local nor non-local effective medium calculations allow discrimination of the emitter position in the unit cell of the array. Therefore, both EMTs provide an estimate of the average lifetime. Nevertheless, the transfer matrix-based mode-matching formalism allows additional averaging of the lifetime over emitter position along the nanorod length.

It is important to underline that both local and non-local effective medium theories predict almost identical transmission and reflection spectra of metamaterials (Supplementary Fig. S2), as the plane wave, incident from vacuum, only weakly couples to high-index additional waves inside the metamaterial. At the same time, point dipoles emitting from within the composite can successfully couple to these high-index modes, enabled by spatial dispersion. In fact, emission into additional waves dominates the decay dynamics while the metamaterial operates in the elliptical regime.

\section{Engineering emission lifetime with metamaterial nonlocality}

Adjusting the geometry and/or composition of a metamaterial opens the door for engineering quantum optical effects inside the composites. This is illustrated in Figure $4 \mathrm{c}$ where the lifetime dynamics in the composites studied above are compared with dynamics of emission in a similar metamaterial with nanorod diameter and unit cell dimension scaled by $50 \%$. It is interesting to note that the reduction of the unit cell does not make the optical response of metamaterial appear more local; i.e., it does not lead to the enhancement of local density of states at ENZ and hyperbolic frequencies. Rather, 3D numerical simulations and non-local effective medium theory predict that the reduction of unit cell size yields a further, order of magnitude, broadband enhancement of decay rate (Figure $4 \mathrm{c}$ and Supplementary Fig. S3c and S3d), despite the local effective medium parameters of the metamaterial being the same. 
In order to check the stability of the non-local EMT approach to the lifetime modification estimations, we have varied the metamaterial geometrical parameters near the nominal parameters of the metamaterial used in the experiments (Supplementary Fig. S6). The obtained lifetime modifications are close to each other with similar spectral variations and all fall within the range of lifetimes predicted by the numerical simulations (Figure $4 \mathrm{~b}$ ).

Note that both non-local EMT and numerical solutions of Maxwell equations predict comparable enhancement of decay rate as a result of the unit cell reduction. As before, the quantitative difference between predictions of non-local EMT and numerical solutions reflects the limitation of an effective medium response, that nevertheless reveals the important physics and provides a relatively fast estimate of the radiation decay enhancement. Numerical solutions of Maxwell equations enable one to calculate the detailed optical response at any location at the expense of computational complexity and time.

\section{Non-local homogenization theory for designs of large-scale hybrid optical sources}

Understanding and predicting light-matter interactions can be drastically improved with the help of adequate homogenization theory. Local effective medium theories, under some conditions, provide an avenue to understand and design transmission and reflection of composite media. However, as shown above, a local EMT drastically fails when applied to calculations of a macroscopic Purcell effect. At the same time, a non-local EMT can be used to calculate Purcell enhancement in nanorod composites. From the fundamental science standpoint, non-local EMT provides a quantitative description lightmater interaction processes inside composite and identifies the dominant decay channels for the molecules, and can be used to engineer these channels. From the applications standpoint, non-local EMT allows one to avoid complex and extremely time- and memoryconsuming numerical solutions of Maxwell equations for applications that rely on macroscopic number of fluorophores, distributed throughout the composite. In applications that require analysis of a position-dependent Purcell effect, numerical solutions of Maxwell equations cannot be avoided.

\section{CONCLUSION}

In summary, spontaneous radiation of an emitter inside nanorod-based metamaterials exhibiting non-local electromagnetic behavior was experimentally demonstrated and analyzed theoretically and numerically. It was shown that the effect of structural non-locality which results in the so-called additional electromagnetic modes in the metamaterial has significant impact on the density of photonic states inside the composite media and, as the result, quantum electrodynamic processes. The modal structure of a non-local composite material has a major impact on spontaneous decay dynamics, setting a fundamental limit on the overall emission rate enhancement and determines its spectral behavior. Careful consideration of structural non-locality allows the design of the density of optical states with geometrical parameters of the metamaterial composite beyond the limitations of the standard effective medium model, thus opening new opportunities for engineering and tailoring quantum optical processes inside metamaterials.

\section{CONFLICT OF INTEREST}

The authors declare no conflict of interest.

\section{ACKNOWLEDGEMENTS}

This work has been funded in part by ESPRC (UK), the ERC iPLASMM project (321268), and the US Army Research Office (Grant No. W911NF-12-1-0533). $\mathrm{AZ}$ acknowledges support from the Royal Society and the Wolfson Foundation. PG acknowledges TAU Rector Grant and German-Israeli Foundation (GIF, grant number 2399). The data access statement: all the data supporting this research are presented in full in the results section and the supplementary information.

1 de Broglie L. XXXV. A tentative theory of light quanta. Philos Mag Lett 2006; 86: 411-423.

2 Einstein A, Podolsky B, Rosen N. Can quantum-mechanical description of physical reality be considered complete? Phys Rev 1935; 47: 777-780.

3 Bell JS. On the problem of hidden variables in quantum mechanics. Rev Mod Phys 1966; 38: 447-452.

4 Freedman SJ, Clauser JF. Experimental test of local hidden-variable theories. Phys Rev Lett 1972; 28: 938-941.

5 Aspect A, Grangier P, Roger G. Experimental realization of einstein-podolsky-rosenbohm gedankenexperiment: a new violation of Bell's inequalities. Phys Rev Lett 1982; 49: 91-94.

6 Agranovich VM, Ginzburg VL. Crystal Optics with Spatial Dispersion, and Excitons 2nd edn. Pergamon Press: New York, USA, 1984.

7 Pollard RJ, Murphy A, Hendren WR, Evans PR, Atkinson R et al. Optical nonlocalities and additional waves in epsilon-near-zero metamaterials. Phys Rev Lett 2009; 102: 127405.

8 Poddubny AN, Belov PA, Ginzburg P, Zayats AV, Kivshar YS. Microscopic model of Purcell enhancement in hyperbolic metamaterials. Phys Rev B 2012; 86: 035148.

9 Lakowicz JR. Principles of Fluorescence Spectroscopy 3rd edn. Springer: USA, 2006.

10 Provencher SW. Inverse problems in polymer characterization: Direct analysis of polydispersity with photon correlation spectroscopy. Die Makromol Chemie 1979; 180: 201-209.

11 Ford GW, Weber WH. Electromagnetic effects on a molecule at a metal surface. Surf Sci 1981; 109: 451-481.

12 Vogel W, Welsch DG. Quantum Optics 3rd edn. Wiley-VCH: Weinheim, Germany, 2006.

13 Ginzburg P. Cavity quantum electrodynamics in application to plasmonics and metamaterials. Rev Phys 2016; 1: 120-139.

14 Wangberg R, Elser J, Narimanov EE, Podolskiy VA. Nonmagnetic nanocomposites for optical and infrared negative-refractive-index media. J Opt Soc Am B 2006; 23: 498-505.

15 Zhukovsky SV, Andryieuski A, Takayama O, Shkondin E, Malureanu R et al. Experimental demonstration of effective medium approximation breakdown in deeply subwavelength all-dielectric multilayers. Phys Rev Lett 2015; 115: 177402.

16 Lissberger PH, Nelson RG. Optical properties of thin film Au-MgF 2 cermets. Thin Solid Films 1974; 21 : 159-172.

17 Wells BM, Zayats AV, Podolskiy VA. Nonlocal optics of plasmonic nanowire metamaterials. Phys Rev B 2014; 89: 035111.

18 Novotny L, Hecht B. Principles of Nano-Optics 2nd edn. Cambridge University Press: Cambridge, UK, 2012.

19 Brueck SRJ. Radiation from a dipole embedded in a dielectric slab. IEEE J Sel Top Quantum Electron 2000; 6: 899-910.

20 Podolskiy VA, Ginzburg P, Wells B, Zayats A. Light emission in nonlocal plasmonic metamaterials. Faraday Discuss 2015; 178: 61-70.

21 Atre AC, Brenny BJM, Coenen T, García-Etxarri A, Polman A et al. Nanoscale optical tomography with cathodoluminescence spectroscopy. Nat Nanotechnol 2015; 10: 429-436.

22 Krishnamoorthy HNS, Jacob Z, Narimanov E, Kretzschmar I, Menon VM. Topological transitions in metamaterials. Science 2012; 336: 205-209.

23 Vasilantonakis N, Wurtz GA, Podolskiy VA, Zayats AV. Refractive index sensing with hyperbolic metamaterials: strategies for biosensing and nonlinearity enhancement. Opt Express 2015; 23: 14329-14343.

24 Jacob Z, Smolyaninov II, Narimanov EE. Broadband Purcell effect: Radiative decay engineering with metamaterials. Appl Phys Lett 2012; 100: 181105.

25 Purcell EM. Spontaneous emission probabilities at radio frequencies. Phys Rev B 1946; 69: 674 .

26 Tumkur TU, Kitur JK, Bonner CE, Poddubny AN, Narimanov EE et al. Control of Förster energy transfer in the vicinity of metallic surfaces and hyperbolic metamaterials. Faraday Discuss 2015; 178: 395-412.

27 Tumkur T, Zhu G, Black P, Barnakov YA, Bonner CE et al. Control of spontaneous emission in a volume of functionalized hyperbolic metamaterial. Appl Phys Lett 2011; 99: 151115

28 Kruk SS, Wong ZJ, Pshenay-Severin E, O'Brien K, Neshev DN et al. Magnetic hyperbolic optical metamaterials. Nat Commun 2016; 7: 11329.

29 Wurtz GA, Pollard R, Hendren W, Wiederrecht GP, Gosztola DJ et al. Designed ultrafast optical nonlinearity in a plasmonic nanorod metamaterial enhanced by nonlocality. Nat Nanotechnol 2011; 6: 107-111. 
30 Tsai KT, Wurtz GA, Chu JY, Cheng TY, Wang HH et al. Looking into meta-atoms of plasmonic nanowire metamaterial. Nano Lett 2014; 14: 4971-4976.

31 Scully MO, Zubairy MS. Quantum Optics. Cambridge University Press: Cambridge, UK, 1997.

32 Bonifacio $\mathrm{R}$, Lugiato LA. Cooperative radiation processes in two-level systems: Superfluorescence. Phys Rev A 1975; 11: 1507-1521.

33 Martín-Cano D, Martín-Moreno L, García-Vidal FJ, Moreno E. Resonance energy transfer and superradiance mediated by plasmonic nanowaveguides. Nano Lett 2010; 10 3129-3134.

34 Slobozhanyuk AP, Ginzburg P, Powell DA, lorsh I, Shalin AS et al. Purcell effect in hyperbolic metamaterial resonators. Phys Rev B 2015; 92: 195127. (c) (i) $\odot$ This work is licensed under a Creative Commons AttributionNC No NonCommercial-NoDerivs 4.0 International License. The images or other third party material in this article are included in the article's Creative Commons license, unless indicated otherwise in the credit line; if the material is not included under the Creative Commons license, users will need to obtain permission from the license holder to reproduce the material. To view a copy of this license, visit http:// creativecommons.org/licenses/by-nc-nd/4.0/

(C) The Author(s) 2017

Supplementary Information for this article can be found on the Light: Science \& Applications' website (http://www.nature.com/lsa). 